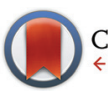

CrossMark

\&lick for updates

Cite this: Polym. Chem., 2015, 6 , 1584

Received 7th November 2014, Accepted 2nd December 2014

DOI: $10.1039 / c 4 p y 01521 \mathrm{e}$

www.rsc.org/polymers

\title{
Novel functionalization of porous polypropylene microfiltration membranes: via grafted poly- (aminoethyl methacrylate) anchored Schiff bases toward membrane adsorbers for metal ions
}

\begin{abstract}
Fatemeh Farjadian, ${ }^{\mathrm{a}, \mathrm{b}}$ Sebastian Schwark ${ }^{\mathrm{a}}$ and Mathias Ulbricht*a
In this work we introduce novel synthetic methods for the modification of a macroporous polypropylene (PP) membrane with poly(2-aminoethyl methacrylate) (polyAEMA) and subsequently anchoring a Schiff base with the aim of adsorbing specific metal ions from aqueous solution. The Schiff base synthesis on the surface of the PP membrane was done by a sequence of reactions. First the hydrophobic character of a commercial PP membrane (pore diameter $0.4 \mu \mathrm{m}$ ) was modified via UV irradiation-induced "graftingfrom" of poly(2-hydroxyethyl methacrylate) (polyHEMA). The hydroxyl groups of polyHEMA were then reacted with the pre-synthesized photoinitiator 4-ethoxy-5-oxo-4,5-diphenylpentanoyl bromide. UVirradiation was thereafter used for the "grafting-from" of polyAEMA. The free amino groups of this grafted comb-like brush layer on the surface were reacted with salicylaldehyde to form a Schiff base. ATR-FTIR spectroscopy was carried out to determine the functional groups' introduction and conversion. Scanning electron microscopy images showed the changes between unmodified and modified membranes. The specific surface area was determined by nitrogen adsorption and BET analysis, and the water permeability was also measured. The efficiency of membrane adsorbers with a Schiff base in the grafted layer in binding to $\mathrm{Cu}(॥)$ ions was determined by atomic absorption spectroscopy. Overall, the established functionalization sequences and the obtained functionality have potential for the development of efficient adsorber materials.
\end{abstract}

\section{Introduction}

Membrane separation is nowadays one of the most important technologies for the treatment of waste water. ${ }^{1-4}$ Microfiltration is an important process which has found application in water treatment. Conventional microfiltration membranes are typically used for their potential capability in the process of separation of (colloidal) particles based on size exclusion or depth filtration. ${ }^{1-4}$ Developing the membrane's functionalization methods would enable the water treatment industries to also remove heavy metals with small sizes that could not be rejected by macropores. One of the most effective techniques which has been developed for this purpose is an adsorption method. The process of adsorption in heavy metal treatment of wastewater involves ion uptake by the active surface of an absorbent. ${ }^{5}$ The adsorbent may be composed of mineral,

\footnotetext{
${ }^{a}$ Lehrstuhl für Technische Chemie II, Universität Duisburg-Essen, Universitätsstr. 5, 45141 Essen, Germany. E-mail: mathias.ulbricht@uni-essen.de;

Fax: +49 0201-1833147; Tel: +49 0201-1833144

${ }^{b}$ Center for Nanotechnology in Drug Delivery, Shiraz University of Medical Sciences, Shiraz 71345, Iran
}

organic or inorganic materials such as silica, synthetic polymers, natural materials and industrial by-products. ${ }^{6-9}$ By introducing complexing agent groups as an adsorbent in a membrane, the capability of membranes for removal of heavy metals could be expanded.

During the past decade, several metal retaining membranes have been developed for efficient removal of heavy metals. The application of polyethyleneimine (PEI) with a large amount of amino and imino groups in its polymer chain has been investigated in membrane synthesis as a chelating agent for $\mathrm{Pb}(\mathrm{II})$, $\mathrm{Cd}(\mathrm{II}), \mathrm{Cu}(\mathrm{II}), \mathrm{Cr}(\mathrm{III})^{10,11}$ and $\mathrm{Hg}$ (II) ion removal. ${ }^{12} \mathrm{~A}$ thiol functionalized mesoporous poly(vinyl alcohol)/ $\mathrm{SiO}_{2}$ composite nanofiber membrane was synthesized and used for $\mathrm{Cu}(\mathrm{II})$ removal. ${ }^{13}$ A metal-complexing membrane containing poly(4vinylpyridine) was prepared and used for removal of $\mathrm{Hg}$ (II) from aqueous solution. ${ }^{14}$ A multifunctional porphyrin membrane was prepared and showed selectivity for $\mathrm{Cd}(\mathrm{II})$ ion adsorption when other cationic species existed in solution. ${ }^{15}$

Schiff bases are among the most widely utilized ligands in metal coordination chemistry and are found to be stable under oxidative and reductive conditions. They are easily formed by the condensation of primary amines and aldehydes and have 
found application as a good chelating ligand anchored polymer for solid support catalysis by different metals. ${ }^{16-18}$ In membrane chemistry, a number of potentiometric sensors based on Schiff bases and their complexes have been reported. ${ }^{19,20}$ Many studies have focused on the use of a Schiff base as a cation carrier in ion-selective electrodes for determining ions such as $\mathrm{Al}($ III) $),{ }^{21} \mathrm{Co}(\mathrm{II}),{ }^{22} \mathrm{Cu}$ (II),${ }^{23} \mathrm{Zn}$ (II), ${ }^{24} \mathrm{Ag}$ (I),${ }^{25} \mathrm{Hg}$ (II) ${ }^{26}$ and also for photovoltaic application. ${ }^{27}$

A typical microfiltration membrane which can be functionalized for further application is made from polypropylene (PP). The surface modification of a hydrophobic membrane can change its characteristic to hydrophilic or make it accessible for further functionalization. ${ }^{28-30}$ A permanent modification by polymer brush layers will be best realized by a covalent grafting to the base polymer via in situ surface-selective copolymerization of functional monomers from the surface. This "grafting-from" approach has proved to be very flexible and versatile. ${ }^{3,32}$ There are several approaches for grafting from surfaces, involving plasma treatment, UV irradiation and ozone methods. ${ }^{31,32}$ UV-initiated graft copolymerization is a powerful technology with high efficiency, a low tendency for base membrane degradation and low cost. It has been used widely for the functionalization of various membranes made of different materials, for example, polyacrylonitrile, ${ }^{33,34}$ polysulfone, ${ }^{35}$ polypropylene ${ }^{28-30}$ or polytetrafluoroethylene. ${ }^{36}$ The first step in UV-initiated "grafting-from" copolymerization is usually the immobilization of a photoinitiator on the polymer surface. Three approaches are typically used for the immobilization of a photoinitiator on the membrane surface: first, physical adsorption, ${ }^{36,37}$ second, an entrapping method, ${ }^{28-30}$ and third, covalent initiator attachment. ${ }^{38}$ The photoinitiator entrapping method as originally proposed for a PP membrane is based on pre-swelling in heptane, subsequent exchange to a polar solvent and thus entrapping of the photoinitiator at the surface layer of the $\mathrm{PP}^{28-30} \mathrm{~A}$ variety of photoinitiators was compared in this method, but benzophenone (BP) was found to be one of the best choices. ${ }^{39}$ The efficiency of this method can be low, especially because of the re-dissolution of the photoinitiator into the monomer solution used in the grafting step. Recently, we reported a novel surface selective photografting method via covalent initiator attachment to the membrane surface followed by "grafting-from" initiated by UVirradiation. ${ }^{38}$ One of the most outstanding advantages of the UV-irradiation method is controlled surface polymerization. Controlling the architecture of the grafted brushes layer is important in order to optimize membrane performance. This goal can be achieved by optimizing grafting parameters such as: the initiator concentration, UV intensity and UV irradiation time in combination with varied monomer mixtures. ${ }^{28-30}$

In continuation of our previous studies on the functionalization of a PP membrane as an adsorbent of a protein ${ }^{29,30}$ and our above mentioned recent study, ${ }^{38}$ we introduce here novel synthetic methods for the modification of a PP membrane with grafted comb-like poly(2-aminoethyl methacrylate) and anchoring a Schiff base in that layer to obtain metal ion membrane adsorbers.

\section{Experimental}

\section{Materials}

Polypropylene (PP) membranes (Accurel PP 2E HF, cut-off pore size $\sim 0.4 \mu \mathrm{m}$, membrane thickness $\sim 150 \mu \mathrm{m}$ ) were purchased from Membrana $\mathrm{GmbH}$, Wuppertal. 2-Aminoethyl methacrylate hydrochloride (AEMA; min. 95\%) was purchased from Polysciences, Inc. $\mathrm{N}$-(2-Aminoethyl) methacrylamide hydrochloride (AEMAm; 98\%) and 2-hydroxyethyl methacrylate (HEMA; 97\%) were purchased from Sigma-Aldrich. The other reagents and solvents were of chemical purity and were obtained from Fluka or Sigma-Aldrich. All chemicals were used as received.

\section{Methods}

Synthesis of the photo-initiator

4-Ethoxy-5-oxo-4,5-diphenylpentanoic acid (1). All details can be found in ref. 40 .

4-Ethoxy-5-oxo-4,5-diphenylpentanoyl bromide (2). All details can be found in ref. 38 .

Primary modification of a PP membrane with poly(2-hydroxyethyl methacrylate) (polyHEMA (I)). The weight of a membrane sample with a diameter of $48 \mathrm{~mm}$ was measured prior to the experiments. Thereafter, it was soaked in a $2 \mathrm{~mL}$ solution of $1 \mathrm{wt} \%$ benzophenone (BP) in heptane for $1 \mathrm{~h}$. Then the membranes were kept in methanol for 10 minutes and directly immersed for 2 hours in $2 \mathrm{wt} \%$ of 2 -hydroxyethyl methacrylate (HEMA) solution. Next, the membranes were immersed in $2 \mathrm{~mL}$ of HEMA solution in a Petri dish $(60 \mathrm{~mm} \times 15 \mathrm{~mm})$. Subsequently, a second smaller Petri dish was placed on the sample, as described in ref. 41. The membrane was irradiated using a high intensity UV lamp at $42 \mathrm{~mW} \mathrm{~cm}^{-2}$ for $6 \mathrm{~min}$. Thereafter, it was washed for $2 \mathrm{~h}$ with pure water and dried in an oven under vacuum at $60{ }^{\circ} \mathrm{C}$ for $2 \mathrm{~h}$ and weighed to determine the degree of graft-polymer modification (DG). DG was calculated according to the following formula:

$$
\mathrm{DG}=\left(W_{1}-W_{0}\right) / W_{0} S_{\text {spec }}
$$

where $W_{0}$ is the initial sample weight, $W_{1}$ is the weight after modification, and $S_{\text {spec }}$ represents the specific surface area of the membrane after each modification step determined by BET analyses ( $c f$. below). The DG after this modification step along with data for the base membrane $\left(S_{\text {spec }}=27.2 \mathrm{~m}^{2} \mathrm{~g}^{-1}\right)$ can be converted to $\left.\mathrm{DG}(\%)\left(\mathrm{DG}(\%)=\left(W_{1}-W_{0}\right) / W_{0} \times 100\right)\right)$ by

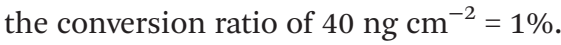

Covalent immobilization of the photo-initiator on primary modified PP with polyHEMA (II). Each membrane modified with polyHEMA was kept in a solution of $40 \mathrm{mg}$ 5-ethoxy-5-oxo4,5-diphenyl-pentanoyl bromide (2) and $0.72 \mathrm{mg}$ benzoyl bromide, in the presence of $5 \mu \mathrm{L}$ triethylamine, in dried acetonitrile, for 12 hours and shaken at $55^{\circ} \mathrm{C}$. After that, samples were washed sequentially with ethanol, water, and ethanol and then dried in an oven under vacuum at $60^{\circ} \mathrm{C}$ for $2 \mathrm{~h}$ and were then weighed.

Secondary comb-like modification of the membrane with polyAEMA (III). Membranes with polyHEMA and the immobi- 
lized photo-initiator were first soaked in methanol for 1 minute, kept in a $6 \mathrm{wt} \%$ solution of AEMA for 30 minutes and then irradiated in a Petri dish containing a monomer solution while the secondary Petri dish was placed on the sample for 15 min using a high intensity UV lamp of $60 \mathrm{~mW}$ $\mathrm{cm}^{-2}$. Next, samples were washed with pure water for $2 \mathrm{~h}$ and then with methanol, dried in an oven under vacuum at $60{ }^{\circ} \mathrm{C}$ for $2 \mathrm{~h}$ and then weighed.

Synthesis of Schiff base modified membrane (V). For this step, a modified membrane containing the comb-like grafted structure polyAEMA should be neutralized, so samples were firstly soaked in methanol and then kept in a solution of $0.2 \mathrm{M}$ $\mathrm{K}_{2} \mathrm{CO}_{3}$ for $1.5 \mathrm{~h}$ and shaken, washed with pure water for $15 \mathrm{~min}$, dried and thereafter weighed (IV). Then samples were kept in a $0.5 \mathrm{M}$ solution of salicylaldehyde in DMF at $60^{\circ} \mathrm{C}$ for $24 \mathrm{~h}$ for Schiff base synthesis. In this step the membrane's color changed from white to yellow. Then the samples were washed sequentially with ethanol, pure water and ethanol for $2 \mathrm{~h}$ and then dried as described before.

All the sequential modification steps (from (I) to (V)) described above were repeated for each modification condition at least three times with independent samples. For membrane functionalization under optimized conditions, a total of 40 samples (V) were prepared which were then used for the analysis of copper binding ( $c f$. below). Average values and standard deviations for DG are reported.

\section{Copper adsorption-desorption process}

Schiff base membranes were weighed and then soaked for $1 \mathrm{~min}$ in ethanol. Thereafter they were put into plastic containers, and $10 \mathrm{ml}$ of a $\mathrm{CuBr}$ solution with different concentrations $\left(C_{0}\right)$ in water were added. Containers were shaken overnight. After that, the solutions were analysed by atomic absorption spectroscopy (AAS) to determine the equilibrium concentration $\left(C_{\text {eq }}\right)$. Membrane samples were then washed with Milli-Q water for $2 \mathrm{~h}$. For the determination of the amount of $\mathrm{Cu}$ adsorbed on the membrane surface, samples were immersed in $10 \mathrm{~mL}$ of a concentrated $\mathrm{HCl}$ solution for 10 hours. Then the solutions were analysed by AAS to determine the amount of adsorbed $\mathrm{Cu}\left(C_{\text {elute }}\right)$. The mass $q$ of copper absorbed at equilibrium per $\mathrm{g}$ of membrane $\left(\mathrm{mg} \mathrm{g}^{-1}\right)$ was obtained as follows:

$$
q=\left(C_{0}-C_{\text {eq }}\right) V / m_{\mathrm{D}}
$$

where $C_{0}$ and $C_{\text {eq }}$ are the initial and equilibrium concentrations of $\mathrm{Cu}(\mathrm{II})$ ions in the solution $\left(\mathrm{mg} \mathrm{L}^{-1}\right)$, respectively. $V$ is the volume of the solution (L) and $m_{\mathrm{D}}$ is the mass of the dry membrane $(\mathrm{g})$.

On the other hand, the mass $q_{\text {elute }}$ of copper desorbed per $g$ of membrane was obtained as follows:

$$
q_{\text {elute }}=C_{\text {elute }} V / m_{\mathrm{D}}
$$

where $C_{\text {elute }}$ is the adsorbed amount of $\mathrm{Cu}(\mathrm{II})$ on the membrane $\left(\mathrm{mg} \mathrm{L}^{-1}\right), V$ is the volume of the solution (L) and $m_{\mathrm{D}}$ is the mass of the dry membrane $(\mathrm{g})$.

All analyses at different $\mathrm{Cu}(\mathrm{II})$ concentrations were done with at least three independent membrane samples, and average values and standard deviations for $q$ values are reported.

\section{Membrane permeability}

An Amicon cell 8100 (Millipore) connected to a reservoir and pressurized with nitrogen was used for all water permeability measurements. All samples obtained from each step were used for measurements. The permeability $P$ is calculated as follows:

$$
P=V / t A p
$$

where $V$ is the volume of water $(\mathrm{L}), t$ is the time for permeation of that volume (h), $A$ the area of the membrane $\left(\mathrm{m}^{2}\right)$, and $p$ the transmembrane pressure (bar).

\section{Instruments and instrumental methods}

Ultraviolet (UV) irradiation was carried out in a self-built UV chamber using a UV-A Print lamp (Hoenle, Gräfelfing, Germany). The membrane surface chemistry was analyzed by Fourier transform infrared spectroscopy attenuated total reflection (FTIR-ATR) using a Bruker Equinox 55 instrument (Bruker Optics Inc., Billerica, MA) equipped with a liquid nitrogen detector. A total of 32 scans were performed at a resolution of $4 \mathrm{~cm}^{-1}$. Scanning electron micrographs and energy dispersive X-ray spectra were obtained using an ESEM instrument, Quanta 400 FEG (FEI, Czech Republic). The membrane samples were dried and sputtered with carbon using a K 550 sputter coater (Emitech, UK). The surface area of the membrane was measured using nitrogen adsorption and analysed with the Brunauer-Emmett-Teller (BET) method on a Beckman Coulter ${ }^{\mathrm{TM}}$ SA3100 instrument. Samples after metal ion loading tests were analyzed with an atomic absorption spectroscopy (AAS) instrument according to DIN EN ISO/lEC 17025:2005. A statistical analysis of data was done using SigmaPlot software version 12 (Systac Software Inc.) for Microsoft Windows. Data were compared using analysis of variance (ANOVA).

\section{Results and discussion}

\section{Membrane functionalization}

Schiff bases containing $-\mathrm{N}=\mathrm{C}$ groups are important chelating agents. The main purpose of this work is to introduce a Schiff base on the surface of macroporous PP membranes for new potential applications in removing heavy metals from water. Toward this aim, a new strategy was designed to achieve the synthesis of a surface anchored Schiff base in a polymer brush layer by a sequence of reactions (Scheme 1).

To synthesize a Schiff base via addition of aldehyde, amino groups should be introduced on the membrane. A direct functionalization using 2-aminoethyl methacrylate hydrochloride (AEMA) or $\mathrm{N}$-(2-aminoethyl) methacrylamide hydrochloride (AEMAm), which both have amino groups as side groups, was performed via entrapping benzophenone as a photoinitiator in the PP membrane surface followed by UV-induced "grafting-from" in aqueous monomer solution. Grafting could 

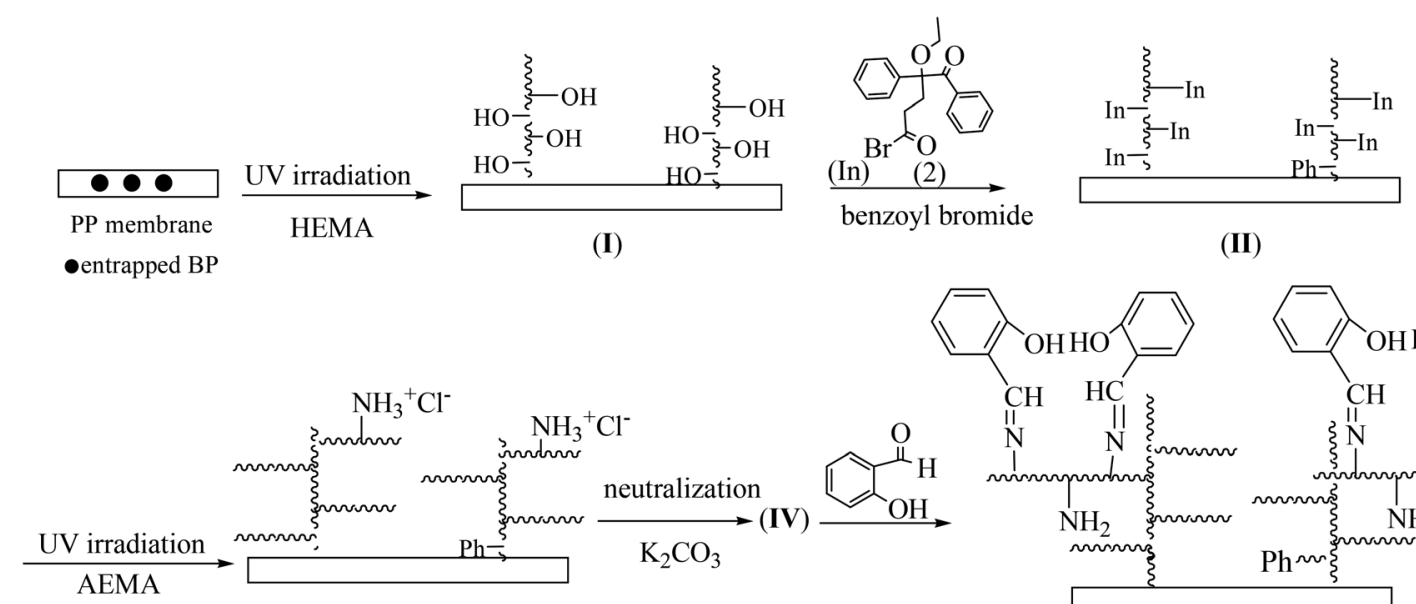

(III)
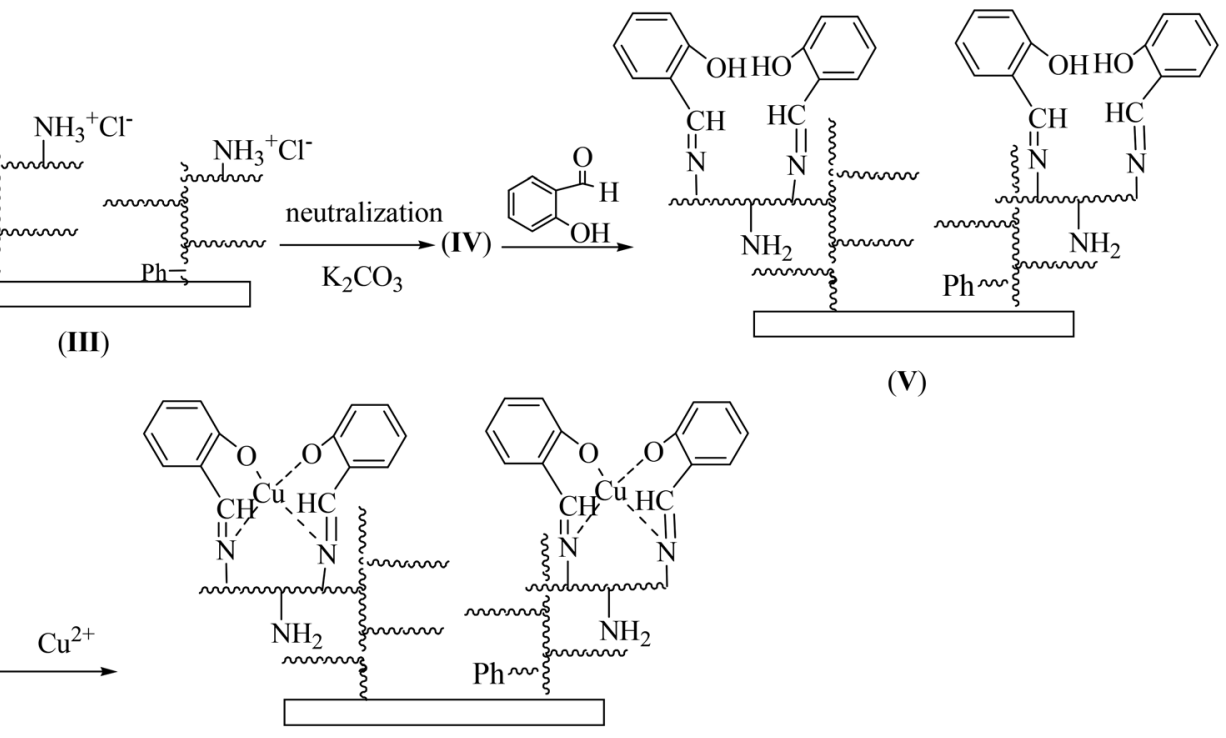

(V)

(VI)

Scheme 1 Synthesis strategy for the modification of the PP membrane toward anchoring copper-chelating Schiff base groups in a comb-like grafted copolymer layer.

be achieved, but there was a decrease in DG after Schiff base synthesis when the membranes were treated with an organic solvent. It is assumed that this happened because graft copolymerization was not the main reaction, and homopolymerization in the monomer solution within the membrane pores had also taken place. Although this procedure for grafting had been proved to be an appropriate method with a minimized extent of homopolymerization, ${ }^{29}$ in this case, homopolymerization might be magnified, due to $\mathrm{H}$ abstraction by UV-excited $\mathrm{BP}$ from the monomer side group yielding starter radical formation. This is probable because the amino-alkyl groups can serve as a co-initiator in combination with the "type II" photoinitiator $\mathrm{BP}^{28-30}$

To overcome the discussed difficulties and achieve high yields of grafting, a new strategy was designed to achieve Schiff base synthesis by a sequence of two "grafting-from" reactions (Scheme 1). These strategies were selected to solve in a first step the side reactivity problem encountered with amino-alkyl monomers like AEMA or AEMAm with a PP membrane using another neutral monomer, and in a second step an alternative ("type I") photoinitiator was used which did not lead to hydrogen abstraction from $\mathrm{CH}_{2}$ segments next to amino groups. A "type I" photoinitiator yields directly starter radicals while a "type II" photoinitiator will react with a co-initiator to produce starter radicals (the latter reaction is the intended reaction of BP with PP).
The selection of monomer was important from two aspects: first, to have hydrophilic character and, second, to have functional groups suitable for covalent attachment of the initiator for the next polymerization, leading to a comb-like grafted structure. For this purpose 2-hydroxyethyl methacrylate (HEMA) was chosen. The surface functionalization by the UVinduced method was done in two steps: (i) entrapping of the photo-initiator (here BP), and (ii) grafting-from of polyHEMA from PP by UV irradiation. In order to achieve a low grafting

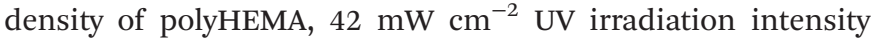
and $1 \mathrm{wt} \%$ initiator concentration and $2 \mathrm{wt} \%$ monomer concentration were selected for grafting. The efficiency of grafting was investigated as a function of varied UV irradiation time (Fig. 1). As is apparent, polyHEMA grafting occurred very well. By prolonging the irradiation, the DG increased. Samples obtained after 15 minutes grafting time were completely shrunken and deformed. We also observed that when the time of membrane soaking in HEMA solution increased under otherwise identical conditions from $1 \mathrm{~h}$ to $12 \mathrm{~h}$, the DG also increased (Fig. 1). One of the probable reasons might be deep immersion of the porous structure of the membrane in the monomer solution due to slow wetting of the pore surface. In this case, shrinking due to too excessive modification occurred after 12 minutes of irradiation. To achieve our purpose, deep penetration of polymer brushes is preferred. The optimized conditions were $12 \mathrm{~h}$ pre-swelling of membrane samples in 


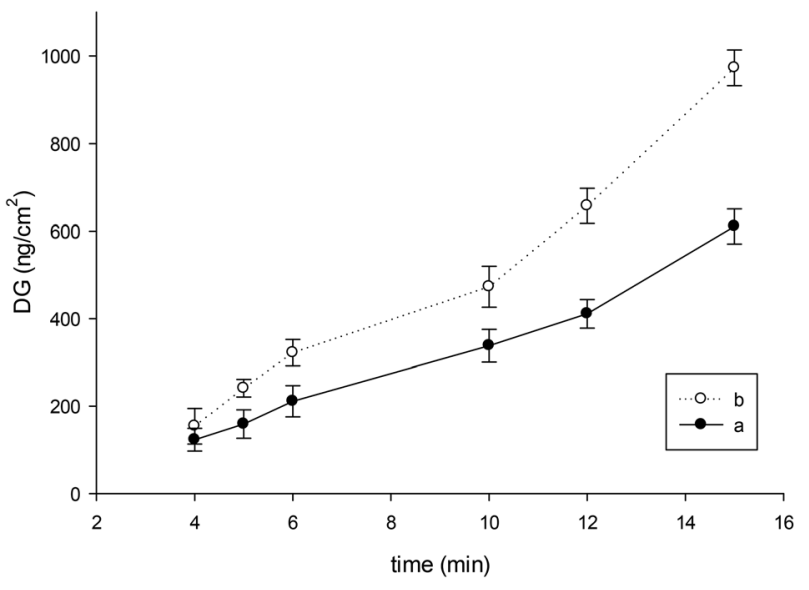

Fig. 1 Influence of UV irradiation time on polyHEMA grafting relative to the specific surface area of the macroporous PP membrane: (a) $1 \mathrm{~h}$, (b) $12 \mathrm{~h}$, soaking in a monomer solution before irradiation.

monomer solution followed by 5 minutes UV irradiation

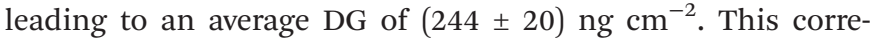
sponds to a relative weight increase of the membrane of $6 \%$.

Successful modification of the PP membrane with polyHEMA enabled us to change the hydrophobic character to hydrophilic and on the other hand, to have hydroxyl groups on the surface. The secondary modification was graft copolymerization of a monomer containing amino group. To inhibit homopolymerization in this step, we covalently immobilized our synthesized initiator 4-ethoxy-5-oxo-4,5-diphenylpentanoyl bromide (2) to hydroxyl groups of polyHEMA of the modified PP membrane. The reaction did not proceed successfully when only compound (2) was used; by gravimetry no changes were observed after this step or during the following UV-irradiation grafting. However, this reaction proceeded successfully in the presence of $20 \%$ of benzoyl bromide (Scheme 2). The calcu-

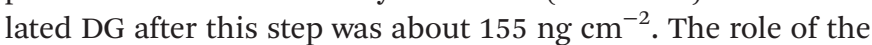
fast reacting benzoyl bromide in this reaction might be that of facilitating the sorption and hence the reaction of the more bulky and hydrophobic compound (2) within the grafted polyHEMA layer.

The mechanism of UV-initiated graft copolymerization is based on a conventional radical mechanism: the more efficient

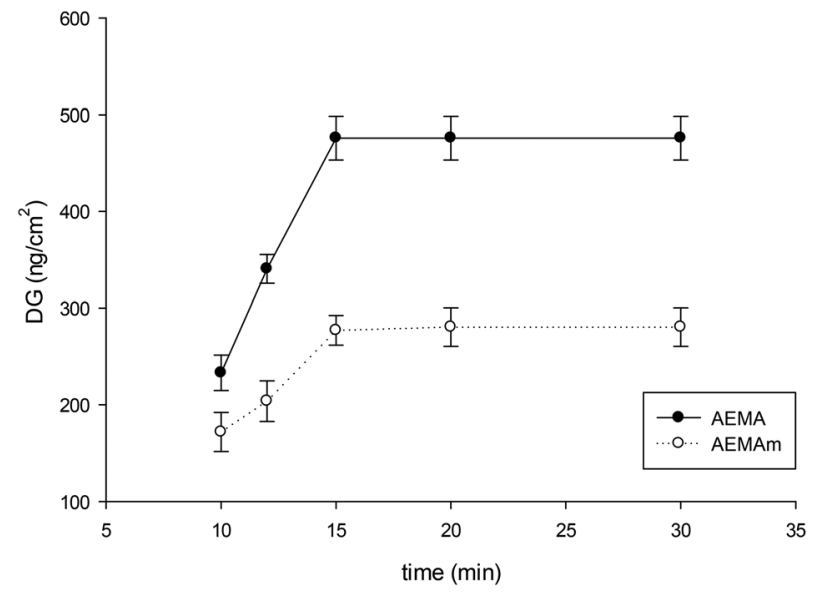

Fig. 2 Influence of UV irradiation time on DG for grafting polyAEMA and polyAEMAm from grafted polyHEMA on the macroporous PP membrane.

starter radical is tethered to the polymer surface ${ }^{32}$ so that the growing polymer chains also become attached to the surface in a comb-like structure on the firstly grafted polyHEMA.

After attachment of (2), the next step was grafting polymer brushes containing amino groups. For this purpose the two monomers AEMA and AEMAm were used. UV-induced grafting-from was performed. Different parameters such as irradiation intensity and time, as well as the monomer concentration, were investigated. The best results were obtained at $60 \mathrm{~mW} \mathrm{~cm}{ }^{-2}$ for UV intensity and $5 \mathrm{wt} \%$ for monomer concentration. The DG changes were investigated with time of UV irradiation, and the results are presented in Fig. 2.

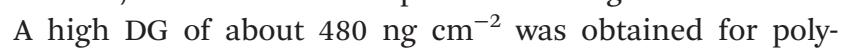
AEMA in 15 minutes of irradiation, but the highest DG for

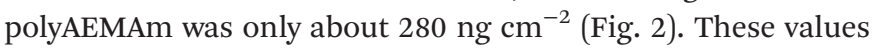
correspond to weight increases of $12 \%$ for grafted polyAEMA and $7 \%$ for grafted polyAEMAm.

We selected AEMA for UV induced grafting with the optimized conditions of $60 \mathrm{~mW} \mathrm{~cm} \mathrm{~cm}^{-2}$ irradiation intensity, $5 \mathrm{wt} \%$ monomer concentration and 15 minutes irradiation time. Membrane samples II were then graft copolymerized with AEMA under optimized conditions to obtain III ( $c f$. Scheme 1). In this case samples III had ammonium salt side groups.

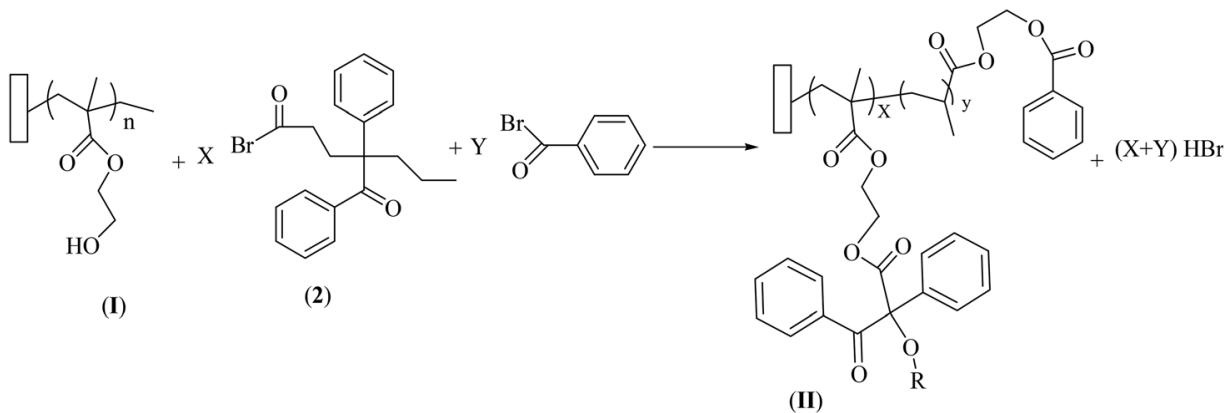

Scheme 2 Synthesis pathway for the immobilization of photo-initiator (2) to the membrane surface. 
To neutralize III, samples were soaked and shaken in $\mathrm{K}_{2} \mathrm{CO}_{3}$ solution to obtain the neutral form IV. The final step was introducing a Schiff base from the amino groups of the grafted layers. Neutralized samples IV were placed in a salicylaldehyde solution for 24 and $48 \mathrm{~h}$ at $55^{\circ} \mathrm{C}$ to obtain $\mathrm{V}$. It seemed that Schiff base formation was complete in $24 \mathrm{~h}$, and prolonging the reaction time did not increase the DG obtained from gravimetric data. After this step the DG change was $225 \mathrm{ng} \mathrm{cm} \mathrm{cm}^{-2}$, which is equal to $50 \%$ conversion of the introduced amino groups (based on DG for that step). This corresponds to a relative weight increase of the membrane of $5.5 \%$.

\section{Characterization of grafted layer structure}

Changing different parameters in the sequential grafting and reaction steps enabled us to obtain the desired grafted layer structure. FTIR-ATR spectra after each step were recorded, and unmodified PP membranes were compared with primary modified I, secondary modified III and Schiff base V. Schiff base membrane (V) was also compared with the Schiff base membrane after $\mathrm{Cu}$ binding (VI) (Fig. 3).

In the spectrum of the primary modified PP membrane with dilute grafting of polyHEMA (I), the stretching absorption peak of the ester carbonyl group appeared with a low intensity at $1727 \mathrm{~cm}^{-1}$. After secondary modification with polyAEMA (III) the stretching absorption band of the ester carbonyl group was more apparent at $1729 \mathrm{~cm}^{-1}$, and for ammonium salt $\left(\mathrm{NH}_{3}^{+}\right)$ a broad band in the range of $3000-3500 \mathrm{~cm}^{-1}$ was observed. In the Schiff base state $(\mathbf{V})$ the changes of the ammonium group to azomethine $(-\mathrm{N}=\mathrm{C}-)$ had resulted in the appearance of a new stretching absorption band at $1634 \mathrm{~cm}^{-1}$. When the Schiff base was treated with copper ions (VI), the complex formation happened and the peak for the $(-\mathrm{N}=\mathrm{C}-)$ group shifted to a lower wavenumber and appeared at $1620 \mathrm{~cm}^{-1}$.

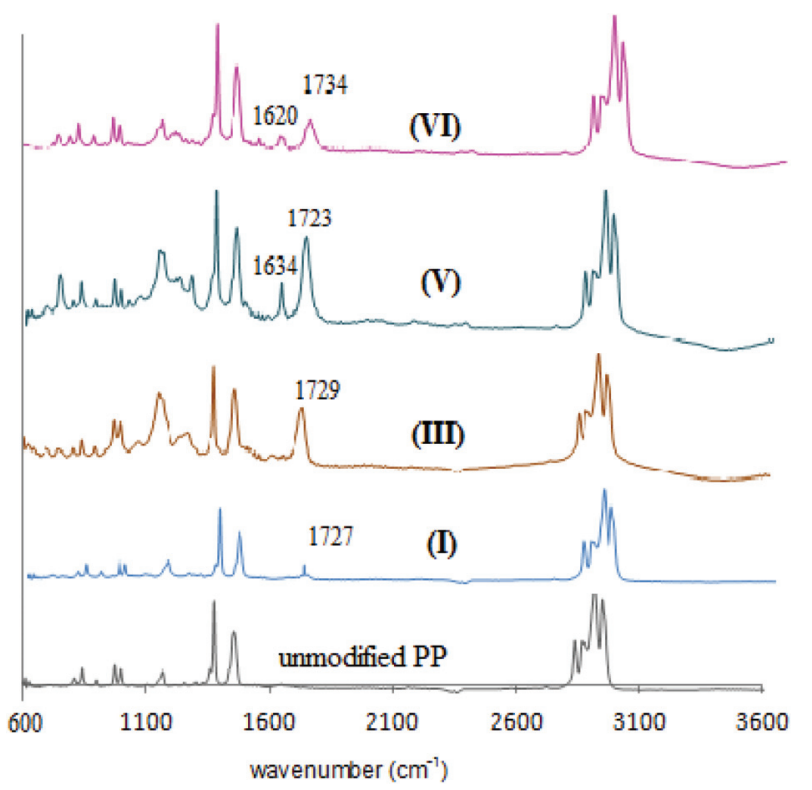

Fig. 3 Comparison of ATR-FTIR spectra for the unmodified PP membrane and modified membranes (I), (III), (V), and (VI) (cf. Scheme 1).
Scanning electron microscopy (SEM) and energy dispersive X-ray (EDX) spectroscopy were carried out for the inner membrane surfaces; images are presented in Fig. 4.

The macroporous structure of the unmodified membrane underwent some significant variations due to functionalization. However, those could only be identified by focusing on details of the cross-section while the overall porous morphology, as seen in Fig. 4( $\left.\mathrm{A}^{\prime}\right)$, remained unchanged. After the primary modification with polyHEMA (I) the membrane pore sizes changed slightly (compare Fig. 4(B) and (A)). However, after secondary modification to yield the comb-like grafted structure (III), the effect of higher DG with polyAEMA (also leading to a more intense IR band; $c f$. Fig. 3) caused that pores seemed to become smaller (compare Fig. 4(C) and (B)). The morphology of the membrane with a Schiff base (V) looked slightly denser in comparison with III (compare Fig. 4(D) and (C)). The presence of copper after using a Schiff base membrane (V) in adsorption experiments was qualitatively proven by the EDX study (data not shown).

The specific surface area of the porous material can be determined by the nitrogen adsorption technique using the BET method. The conventional macroporous membrane (pore size $\geq 50 \mathrm{~nm}$ ) has normally a low specific area. The BET surface area of the unmodified PP membrane was measured to be $27.2 \mathrm{~m}^{2} \mathrm{~g}^{-1}$; this value showed a decreasing trend after each modification step. For primary modified membrane (I) it was $26.8 \mathrm{~m}^{2} \mathrm{~g}^{-1}$, after secondary modification (III) it was $25.3 \mathrm{~m}^{2}$ $\mathrm{g}^{-1}$ and for the Schiff base membrane (V) it was $22.1 \mathrm{~m}^{2} \mathrm{~g}^{-1}$. This observation completely correlates with changes in SEM images (Fig. 4) and the reason is the filling of small pores in the dry state by a grafted polymer.

\section{Water permeability}

The water permeability of unmodified and modified membranes was measured. For a microfiltration membrane this is an important criterion for its performance. The modification resulted in a decrease of pore size and consequently the water permeability decreased with an increase of DG value by each step of modification (Fig. 5).

After the first step of polyHEMA photografting with a DG of about $245 \mathrm{ng} \mathrm{cm}{ }^{-2}$, a permeability of about $11100 \pm 100 \mathrm{~L} \mathrm{~m}^{-2}$ $\mathrm{h}$ bar was obtained. This was only slightly smaller than the data for the unmodified membrane. After the second step, the decrease of permeability was more apparent; a higher amount of grafting ( $\mathrm{DG}=480 \mathrm{ng} \mathrm{\textrm {cm } ^ { - 2 }}$ ) resulted in a water permeability of $9300 \pm 150 \mathrm{~L} \mathrm{~m}^{-2} \mathrm{~h}$ bar. Only relatively small further changes were observed for the Schiff-base membrane (V); permeability was $8600 \pm 150 \mathrm{~L} \mathrm{~m}^{-2} \mathrm{~h}$ bar, in agreement with a DG change of about $225 \mathrm{ng} \mathrm{cm}{ }^{-2}$. Overall, the change of water permeability correlates with DG variation in each step of the modification ( $c f$. Fig. 5), and this is in agreement with the reduction in average pore diameter evoked above.

\section{Adsorption capability of the Schiff base membrane for copper ions}

The behavior of the Schiff base functionalized membrane (V) in $\mathrm{Cu}$ removal was investigated. Membrane samples were put 

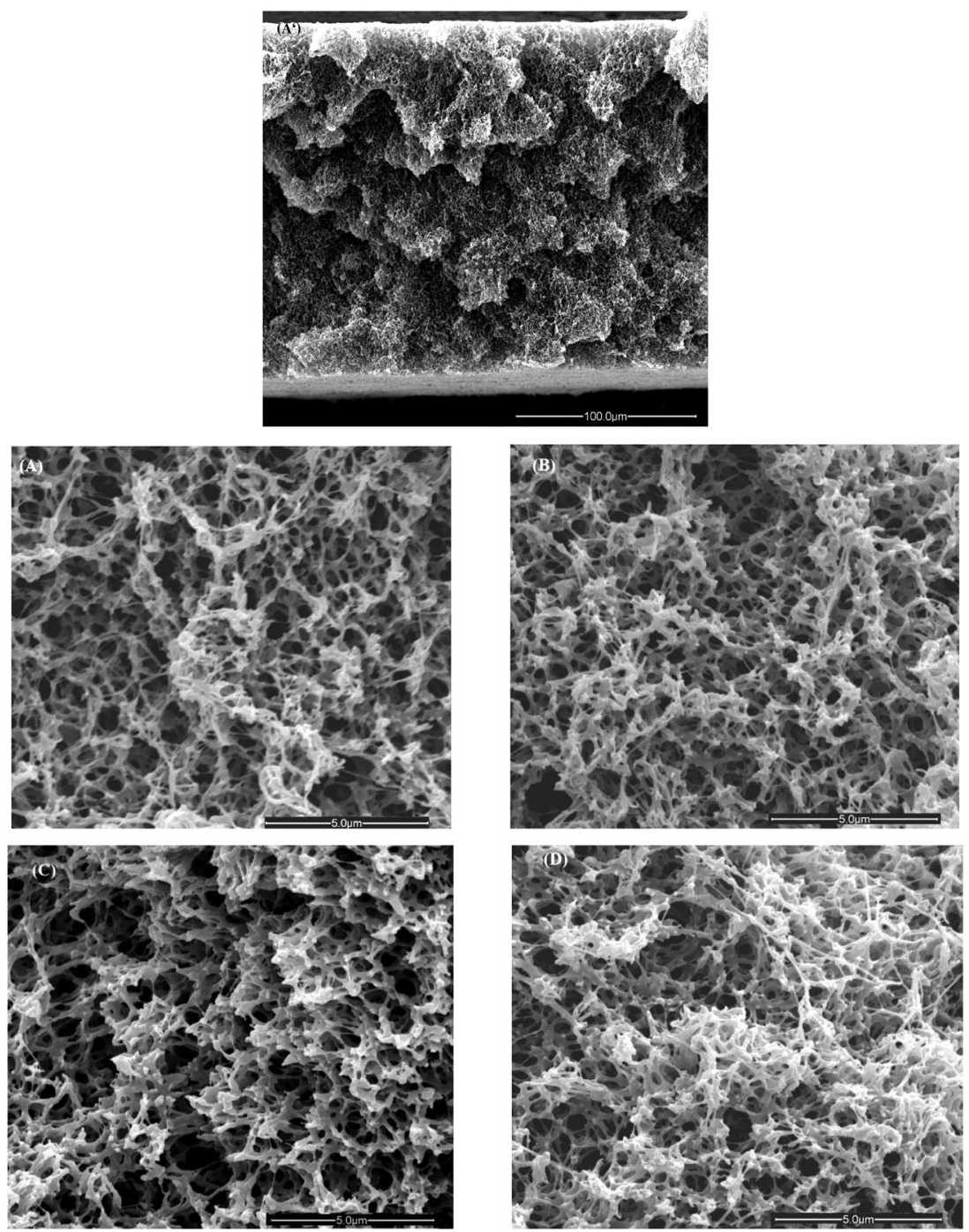

Fig. 4 SEM images: unmodified PP membrane: overview on cross-section ( $\left.A^{\prime}\right)$ and details of cross-section (A); details of cross-section for membranes primary modified with polyHEMA (B), secondary modified with polyAEMA (C), and after the Schiff base functionalization (D).

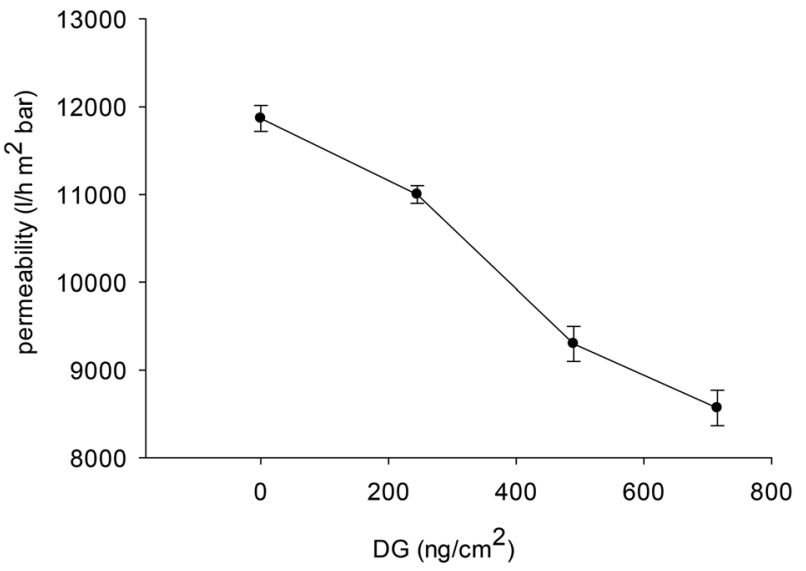

Fig. 5 Effect of DG obtained in the step-wise modification on the water permeability of the membranes. into aqueous $\mathrm{CuBr}$ solution with different concentrations. The adsorbed amount of $\mathrm{Cu}$ on the membrane was calculated from the mass balance, and the results are the mass $q$ of copper adsorbed at equilibrium per gram of membrane $\left(\mathrm{mg} \mathrm{g}^{-1}\right)$ as a function of the equilibrium concentration $C_{\text {eq }}$ of $\mathrm{Cu}$ (II) when the initial concentration $C_{0}$ varied in the range of 2.5 to $2500 \mathrm{mg} \mathrm{L}^{-1}$. The values of $q$ increased with increasing $C_{0}$, as expected if the sorption equilibrium was simply driven by the increasing amount of $\mathrm{Cu}(\mathrm{II})$ in solution.

In order to optimize the use of adsorbents, it is important to understand the adsorption in more detail. Langmuir and Freundlich isotherms are two common models to describe the behavior of adsorbents and were used to analyze the experimental data. ${ }^{42}$ The Langmuir isotherm model which characterizes a homogeneous system in single layer adsorption is often given as follows:

$$
q=q_{\mathrm{m}} K_{\mathrm{L}} C_{\mathrm{eq}} /\left(1+K_{\mathrm{L}} C_{\mathrm{eq}}\right)
$$


where $q$ is as defined before, $q_{\mathrm{m}}$ is the maximum uptake capacity $\left(\mathrm{mg} \mathrm{g}^{-1}\right), C_{\mathrm{eq}}$ is the equilibrium concentration of the adsorbate (here, copper ions) in solution $\left(\mathrm{mg} \mathrm{L}^{-1}\right)$, and $K_{\mathrm{L}}$ is the Langmuir adsorption constant $\left(\mathrm{L} \mathrm{mg}^{-1}\right)$, reflecting the affinity of the absorbate to the absorbent.

The Freundlich isotherm model, which is an empirical equation used to describe a heterogeneous adsorption system, usually in multiple layer adsorption, is often given as follows:

$$
q=K_{\mathrm{f}} C_{\mathrm{eq}}^{1 / n}
$$

where $q$ and $C_{\mathrm{eq}}$ have the same definitions as before and $K_{\mathrm{f}}$ is the Freundlich constant $\left[\left(\mathrm{mg} \mathrm{g}^{-1}\right)\left(\mathrm{L} \mathrm{mg}^{-1}\right)\right]^{1 / n}$, which has a positive correlation with the adsorption capacity, and $n$ is an empirical parameter, in general larger than 1, and it may increase on increasing the degree of heterogeneity.

The fitting of the Langmuir and Freundlich models to the adsorption data of copper ions on the Schiff base membrane (V) was carried out and the results are shown in Fig. 6. We found that best fitting is for a lower concentration range of 2.5 to $250 \mathrm{mg} \mathrm{\textrm {L } ^ { - 1 }}$. The corresponding fitting parameters for the Langmuir and Freundlich isotherm models from the analysis are given in Table 1 . The Freundlich model gave only a very poor fit of the data (low $R^{2}$ ). From the Langmuir model fitting $\left(R^{2}=0.997\right)$, the maximum adsorption capacity of copper ions on $\mathbf{V}$ is predicted to be about $90 \mathrm{mg}^{-1}$.

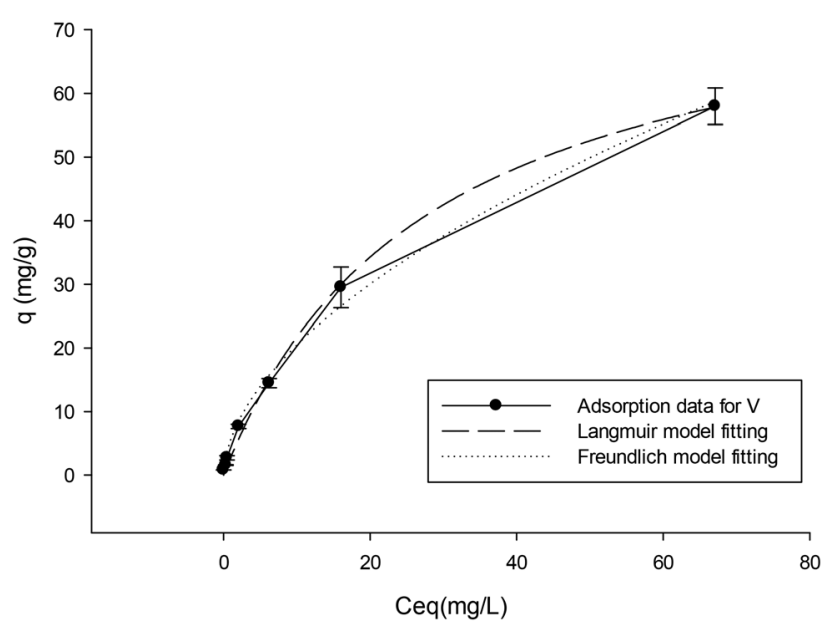

Fig. 6 Adsorption isotherm data and the fitted results of the Langmuir and Freundlich isotherm models.

Table 1 The fitting parameters of the Langmuir and Freundlich isotherm models to the adsorption data of $\mathrm{Cu}(\mathrm{II})$

\begin{tabular}{|c|c|c|c|c|c|c|}
\hline & \multicolumn{3}{|c|}{ Langmuir model } & \multicolumn{3}{|c|}{ Freundlich model } \\
\hline & $\begin{array}{l}q_{\mathrm{m}} \\
\left(\mathrm{mg} \mathrm{g^{-1 }}\right)\end{array}$ & $\begin{array}{l}K_{\mathrm{L}} \\
\left(\mathrm{L} \mathrm{mg}^{-1}\right)\end{array}$ & $R^{2}$ & $n$ & $\begin{array}{l}K_{\mathrm{f}}\left[\left(\mathrm{mg} \mathrm{g}^{-1}\right)-\right. \\
\left.\left(\mathrm{L} \mathrm{mg}^{-1}\right)\right]^{1 / n}\end{array}$ & $R^{2}$ \\
\hline $\begin{array}{l}\text { Adsorption } \\
\text { data of } \mathbf{V}\end{array}$ & 90.3 & 0.03 & 0.996 & 1.78 & 5.3 & 0.996 \\
\hline $\begin{array}{l}\text { Desorption } \\
\text { data of } \mathbf{V}\end{array}$ & 35.7 & 0.006 & 0.723 & 3.63 & 4.1 & 0.942 \\
\hline
\end{tabular}

To obtain a better understanding of membrane $\mathbf{V}$ binding behavior, after metal binding and washing using water at the same $\mathrm{pH}$ as that used in the binding step, the desorption data of $\mathrm{Cu}$ obtained by elution with concentrated $\mathrm{HCl}$ solution was also analyzed. The fitting of the Langmuir and Freundlich models to the desorption data of copper ions strongly bound to the Schiff base membrane (V) was carried out and the results are shown in Fig. 7. The corresponding fitting parameters are given in Table 1.

The results show that the Freundlich model gives a better description of the isotherm based on desorption data. The adsorption heterogeneity calculated as the parameter $n$ for $\mathbf{V}$ is 3.6. This shows the high affinity of $\mathrm{Cu}$ ions to the membrane. The isotherm based on desorption data after washing off weakly bound $\mathrm{Cu}$ shows bimodal behavior. As is apparent, there is a good fitting to the Langmuir model for data with $q_{\mathrm{el}}$ varying in the range of 1.3 to $10 \mathrm{mg} \mathrm{g}^{-1}$. This may be described by theoretical calculation of $q$ for Schiff base ligands in which each $\mathrm{Cu}^{2+}$ chelates with two Schiff base groups (Scheme 3, left side of the cartoon), and the maximum absorbed mass of $\mathrm{Cu}$ at saturation is $10 \mathrm{mg} \mathrm{g}^{-1}$. Since the Langmuir model describes a single mode adsorption, this good correlation can be well understood.

For desorption data with $q$ varying in the range 10 to $28 \mathrm{mg} \mathrm{g}^{-1}$, the isotherm behavior changed. This is due to the

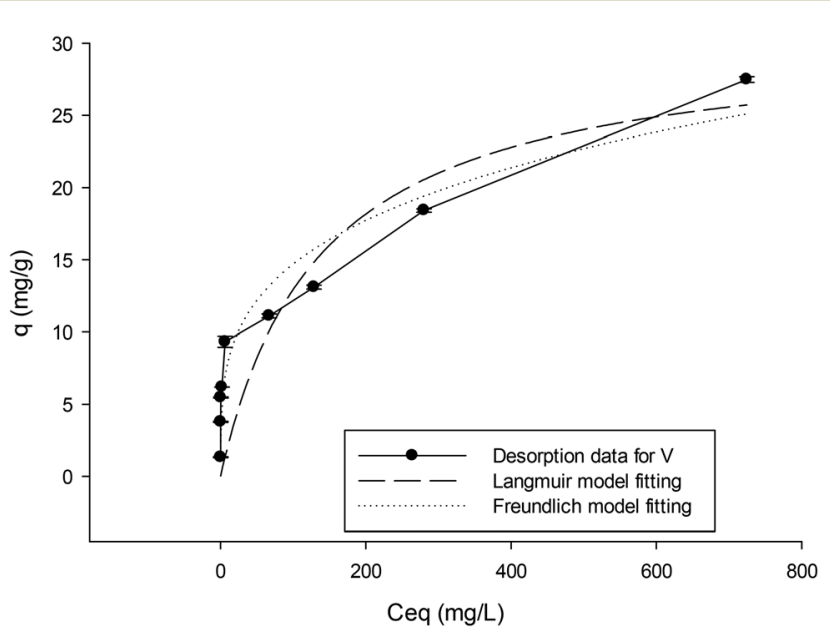

Fig. 7 Adsorption isotherm data from desorption of $\mathrm{Cu}$ after binding and washing and the fitted results of the Langmuir and Freundlich isotherm models.

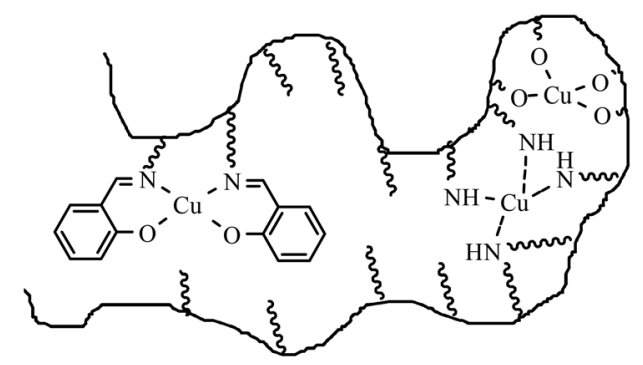

Scheme 3 Schematic illustration of copper binding to chemical groups in a grafted functional polymer layer on the membrane pore surface. 
interference of other functional groups $\left(-\mathrm{NH}_{2},-\mathrm{OH}\right)$ from polyAEMA and polyHEMA brushes in adsorbing $\mathrm{Cu}$ ions (Scheme 3, right side of the cartoon). If we consider that the Schiff base and amino groups (each $\mathrm{Cu}^{2+}$ chelates with four amino groups) interfere, the theoretical amount of $q$ calculated from the DG value of $\mathbf{V}$ would be $20 \mathrm{mg} \mathrm{g}^{-1}$. This description could be the reason for the better fitting of the whole isotherm to the Freundlich model, describing multiple mode adsorption.

\section{Conclusion}

In this study, novel synthetic methods for the functionalization of a PP membrane with comb-like grafted polyAEMA on grafted polyHEMA and subsequently anchoring Schiff base groups were introduced. Modification of the PP membrane was performed using two sequential UV-irradiation methods, by "entrapping" and covalently immobilizing two types of photo-initiators. PolyHEMA was grafted from the PP membrane by entrapping benzophenone as a "type II" photoinitiator. It was shown that a long immersion of the PP membrane in a HEMA solution resulted in a higher DG of grafting within a shorter UV time. A novel method for the immobilization of a "type I" photo-initiator used as a carboxylic acid bromide derivative by covalent attachment to hydroxyl groups of polyHEMA lead to successful grafting of polyAEMA. It was found that the highest degree of grafting without macroscopic deterioration of the membrane integrity was obtained at $60 \mathrm{~mW} \mathrm{~cm}$ c $^{-2}$ UV intensity and $5 \mathrm{wt} \%$ monomer concentration. A Schiff base synthesis was done after neutralization, with the best results obtained using salicylaldehyde solution in DMF and shaking for 24 hours. Successful modifications after each step were proved by ATR-FTIR spectroscopy. Morphological changes were followed by SEM. BET data proved the decrease of the specific surface area in the dry state after each modification which is due to the grafted polymer filling the pore structure. The effect of the grafted layer and chemical modification was investigated by water permeability, which showed good correlation with stepwise degree of grafting increases. The behavior of the Schiff base modified membrane with respect to metal ion capturing was evaluated. From the sorption-desorption isotherms it was found that the modified membrane shows bimodal behavior in metal capturing. In the range of low concentration of $\mathrm{Cu}$, binding to Schiff base groups dominates, and at higher concentration, binding to amino and hydroxyl groups interferes. Consequently, the isotherm from $\mathrm{Cu}$ desorption after washing off the weakly bound metal showed good fitting to the Freundlich model, describing multiple layer adsorption. The permeability of the membrane after the last modification step was still high, which later allowed the use of the membranes in flow-through metal capturing. Overall, the established functionalization sequences and the obtained functionality have significant potential for the development of efficient membrane adsorbers.

\section{Acknowledgements}

F. F. would like to thank the Iran Ministry of Sciences, Research and Technology for supporting her scholarship for visiting Universität Duisburg-Essen. The contribution of the members of staff of Lehrstuhl für Technische Chemie II, Universität Duisburg-Essen, is also acknowledged.

\section{References}

1 A. G. Fane, C. Y. Tang and R. Wang, Membrane Technology for Water: Microfiltration, Ultrafiltration, Nanofiltration, and Reverse Osmosis, ed. P. Wilderer, Treatise on Water Science, Academic Press, Oxford, 2011, pp. 301-335.

2 M. Ulbricht, Polymer, 2006, 47, 2217.

3 T. M. Pendergast and E. M. V. Hoek, Energy Environ. Sci., 2011, 4, 1946.

4 B. Van der Bruggen, C. Vandecasteele, T. Van Gestel, W. Doyen and R. Leysen, Environ. Prog., 2003, 22, 46.

5 M. Suzuki, Adsorption Engineering, Elsevier, Amsterdam, 1990.

6 T. A. Kurniawan, G. Y. S. Chan, W. H. Lo and S. Babel, Sci. Total Environ., 2005, 366, 409.

7 M. A. Barakat, Arabian J. Chem., 2011, 4, 361.

8 F. Fu and Q. Wang, J. Environ. Manage., 2011, 92, 407.

9 W.-S. Wan Ngah and M. A. K. M. Hanafiah, Bioresour. Technol., 2008, 99, 3935.

10 L. Lebrun, F. Vallée, B. Alexandre and Q. T. Nguyen, Desalination, 2007, 207, 9.

11 H. Bessbousse, T. Rhlalou, J. F. Verchére and L. Lebrun, J. Membr. Sci., 2008, 307, 249.

12 H. Bessbousse, T. Rhlalou, J. F. Verchére and L. Lebrun, J. Membr. Sci., 2008, 325, 997.

13 S. Wu, F. Li, H. Wang, L. Fu, B. Zhang and G. Li, Polymer, 2010, 51, 6203.

14 H. Bessbousse, T. Rhlalou, J. F. Verchére and L. Lebrun, J. Phys. Chem. B, 2009, 113, 8588.

15 L. Zhang, Y. H. Zhao and R. Bai, J. Membr. Sci., 2011, 379, 69.

16 K. C. Gupta, A. K. Sutar and C. C. Lin, Coord. Chem. Rev., 2009, 253, 1926.

17 R. Drozdzak, B. Allaert, N. Ledoux, I. Dragutan, V. Dragutan and F. Verpoort, Coord. Chem. Rev., 2005, 249, 3055.

18 B. Tamami and S. Ghasemi, Appl. Catal., A, 2011, 393, 242.

19 F. Faridbod, M. R. Ganjali, R. Dinarvand, P. Norouzi and S. Riahi, Sensor, 2008, 8, 1645.

20 W. Al Zoubi and N. Al Mohanna, Spectrochim. Acta, Part A, 2014, 132, 854.

21 A. Abbaspour, A. R. Esmaeilbeig, A. A. Jarrahpour, B. Khajeh and R. Kia, Talanta, 2002, 58, 397.

22 M. Ghiaci, B. Rezaei, Z. Sadeghi and J. Safaei-Ghomi, J. Chem. Eng. Data, 2010, 55, 2792.

23 M. R. Ganjali, M. Golmohammadi, M. Yousefi, P. Norouzi, M. Salavati-Niasari and M. Javanbakht, Anal. Sci., 2003, 19, 223. 
24 M. Shamsipur, M. Yousefi, M. Hosseini, M. R. Ganjali, H. Sharghi and H. Naeimi, Anal. Chem., 2001, 73, 2869.

25 M. E. M. Hassouna, S. A. A. Elsuccary and J. P. Graham, Spectrochim. Acta, Part B, 2010, 146, 79.

26 M. H. Mashhadizadeh and I. Sheikhshoaie, Talanta, 2003, 60, 73.

27 A. W. Jeevadason, K. K. Murugavel and M. A. Neelakantan, Renewable Sustainable Energy Rev., 2014, 36, 220.

28 A. H. M. Yusof and M. Ulbricht, Desalination, 2006, 200, 462.

29 A. H. M. Yusof and M. Ulbricht, J. Membr. Sci., 2008, 311, 294.

30 M. Ulbricht and H. Yang, Chem. Mater., 2005, 17, 2622.

31 K. Kato, E. Uchida, E. T. Kang, Y. Uyama and Y. Ikada, Prog. Polym. Sci., 2003, 28, 209.

32 D. He, H. Susanto and M. Ulbricht, Prog. Polym. Sci., 2009, 34, 62 .
33 M. Ulbricht, H. Matuschewski, A. Oechel and H. G. Hicke, J. Membr. Sci., 1996, 115, 31.

34 X. Liu, H. Liu, H. Ma, C. Cao, M. Yu, Z. Wang, B. Deng, M. Wang and J. Li, Ind. Eng. Chem. Res., 2012, 51, 15089.

35 M. Ulbricht, M. Riedel and U. Marx, J. Membr. Sci., 1996, 120, 239.

36 G. Z. Liang, T. L. Lu, X. Y. Ma, H. X. Yan and Z. H. Gong, Polym. Int., 2003, 52, 1300.

37 C. Geismann, A. Yaroshchuk and M. Ulbricht, Langmuir, 2007, 23, 76.

38 S. Schwark and M. Ulbricht, Eur. Polym. J., 2012, 48, 1914.

39 W. T. Yang and B. Ranby, Eur. Polym. J., 1999, 35, 1557.

40 H. G. Heine and H. Rudolph, Liebigs Ann. Chem., 1971, 754, 28.

41 H. Susanto, M. Balakrishnan and M. Ulbricht, J. Membr. Sci., 2007, 288, 157.

42 Y. S. Ho, Pol. J. Environ. Stud., 2006, 15, 81. 